\title{
Relación Entre La Intención De La Elección De La Carrera De Ingeniería Civil Y La Iniciativa Emprendedora De Los Estudiantes De Una Institución De Educación Superior Tecnológica En Hidalgo, México ${ }^{1}$
}

\author{
García-Hernández, Yessica \\ Mendoza-Saldivar, Isabel \\ Romo-Medellin, Leiry Desireth
}

Instituto Tecnológico Superior del Oriente del Estado de Hidalgo, Mexico

Doi: 10.19044/esj.2017.v13n34p101 URL:http://dx.doi.org/10.19044/esj.2017.v13n34p101

\begin{abstract}
In the last years, entrepreneurship has generated a strong interest like potential generator of opportunities and professional growth and economics being the Institutions of Higher Education (IES), the link to transmit in the students the competences and skills required that help to boost the enterprising activity. The objective of this paper is to analyze the intention of the selection of a Civil Engineering degree from students of an Institution of Higher Technological Education in Hidalgo, Mexico, based on the Theory of Planned Behavior, with the goal to identify its relationship with the Degree of Entrepreneurship. The GUESSS instrument adaptation was applied, a sample of $n=198$ students. The type of research in quantitative approach, descriptive and relational scope. The results showed that the intention to choose the career to undertake at the end of their studies is $67.7 \%$, increasing to $81.8 \%$ five years after concluding their studies. Regarding the scales that determine the Entrepreneurship Initiative, the one that has a greater perception of the media on the part of the students is Entrepreneurial Intent and the one of low Score is Control of Perceived Behavior. Finally, Pearson's Chi-square value indicates a result of 0.021 which defines that exist a dependency relationship between the students' career choice at the end of their studies and the Degree of Entrepreneurship.
\end{abstract}

\footnotetext{
${ }^{1}$ Se agradece la colaboración en la realización del presente trabajo de investigación a los alumnos de Ingeniería en Gestión Empresarial Diego Hernández Hernández y Sandra Chávez Moreno de Ingeniería Civil, estudiantes del Instituto Tecnológico Superior del Oriente del Estado de Hidalgo.
} 
Keywords: Entrepreneur, Entrepreneurship Initiative, IES, Entrepreneurial Intent

\section{Resumen}

En los últimos años, el emprendimiento ha generado un fuerte interés como potencial generador de oportunidades y crecimiento profesional y económico siendo las Instituciones de Educación Superior (IES), el vínculo para transmitir en los estudiantes las competencias y habilidades necesarias que ayuden a potenciar la actividad emprendedora. El presente trabajo tiene como objetivo analizar la intención de elección de la carrera de Ingeniería Civil de los estudiantes de una Institución de Educación Superior Tecnológica en Hidalgo, México, con base en la Teoría del Comportamiento Planificado, con la finalidad de identificar su relación con el Grado de Iniciativa Emprendedora. Se aplicó la adaptación del instrumento GUESSS, a una muestra de $n=198$ estudiantes. El tipo de investigación es de enfoque cuantitativo, alcance descriptivo y relacional. Los resultados muestran que la intención de elegir la carrera para emprender al termino de sus estudios es de $67.7 \%$ incrementándose a $81.8 \%$ cinco años después de concluida su carrera. Respecto a las escalas que determinan la Iniciativa Emprendedora, la que tiene una mayor percepción media por parte de los estudiantes es Intención Emprendedora y la de menor puntuación es Control de Comportamiento Percibido. Finalmente, el valor de Chi-cuadrado de Pearson, indica un resultado de 0.021 lo cual define que existe una relación de dependencia entre la elección de la carrera de los estudiantes al termino de sus estudios y el Grado de Iniciativa Emprendedora.

Palabras-clave: Emprendedor, Iniciativa emprendedora, IES, Intención emprendedora

\section{Introducción}

Es indiscutible, la gran aportación a la dinámica económica que hoy en día generan los emprendedores, quienes constituyen la base de la pirámide empresarial, debido a que están en proceso de crear, desarrollar o consolidar una empresa (Secretaría de Economía, 2017).

En tal sentido, las Instituciones de Educación Superior (IES) juegan un papel fundamental como promotores del emprendimiento, al respecto Kantis, Federico e Ibarra (2014) mencionan que en el caso de México se presenta una disminución del capital humano emprendedor, la cultura emprendedora, así como de la percepción de las políticas referentes al tema, por otra parte, señalan que es momento de emprender para fomentar la economía, razón por la cual resulta necesario analizar el papel de la 
institución razón de estudio, para trabajar en que se eliminen las debilidades descritas.

Al respecto Jaime, Gómez y Ayala (2016) indican que en México existen 241 programas de estudio disponibles para realizar estudios de licenciatura en el área de ingeniería civil, con una población total de 64,885 alumnos. De estos programas 46 se encuentran acreditados por CACEI a nivel nacional hasta febrero de 2016. En Hidalgo se cuenta con siete programas y una población total de 1,654 alumnos. Sin embargo, debe verificarse la calidad de los mismos y su operación, es decir, la forma en que se imparten las clases, la cobertura de los temas de los programas, la calidad de la impartición y la experiencia y diversidad de formación de los docentes, además de la motivación emprendedora de dichas instituciones (CACEI, 2016).

De acuerdo con lo anterior, un reto más para las Instituciones de Educación Superior en México es analizar los posibles escenarios futuros, esto es, qué condiciones podrán presentarse en la oferta y demanda tanto de estudiantes como de profesionistas de ingeniería pues éstos deben tener capacidad de reacción para afrontar los cambios y no sólo satisfacer la demanda sino hacerlo con profesionales de calidad. Es notable hacer mención que los ingenieros civiles mexicanos se vinculan con colegas de países de América Latina, a través de las empresas que han construido obras en esa región. Aunado a lo anterior, es importante considerar que uno de los retos del siglo XXI de la Ingeniería Civil es impulsar la carrera y generar nuevas oportunidades, fomentando la generación de servicios integrales y estimulando que el estudiante sea emprendedor, trabaje en equipo, haga uso de los desarrollos tecnológicos y de la informática, promoviendo un papel activo en su formación (Jaime et al., 2016).

Sin embargo, una problemática es que los estudios realizados al momento, han centrado su atención en los estudiantes de las áreas administrativa (Delgado, Gómez, Romero y Vázquez, 2008). De igual forma, una situación recurrente en los ingenieros civiles es la aportación en la solución de problemas nacionales y su incorporación a la cadena de valor en la industria. Es entonces, donde el enfoque actual de la carrera de Ingeniería Civil puede dar solución a las problemáticas del momento de manera poco menos que satisfactoria. Por lo anterior, surge la necesidad de un cambio en la carrera que permita generar nuevas oportunidades, para ello se requiere de fomentar la generación de servicios integrales de ingeniería, así como estimular el que el estudiante sea emprendedor, trabaje en equipo, haga uso de los desarrollos tecnológicos y de la informática.

De igual forma, es necesario mencionar que aunque a nivel nacional la opción de emprender, es un reto de la carrera de Ingeniería Civil, la retícula de la institución razón de estudio, no cuenta con asignaturas 
enfocadas hacia el desarrollo de un plan de negocios, también, al momento la institución no cuenta con un diagnóstico que proporcione información sobre la intención de emprender de sus estudiantes, con la finalidad de establecer estrategias que puedan contribuir a la consolidación de esta oportunidad de carrera. Lo anterior, refleja la importancia de estudiar la Iniciativa Emprendedora en estudiantes de Ingeniería Civil, con la intención de conocer la situación actual y trabajar en la disminución de debilidades y maximización de fortalezas, considerando el potencial emprendedor con el que se cuenta. Es así, como la investigación tiene como objetivo, analizar la intención de elección de la carrera de Ingeniería Civil de los estudiantes de una Institución de Educación Superior Tecnológica en Hidalgo, México, con base en la Teoría del Comportamiento Planificado, con la finalidad de identificar su relación con el Grado de Iniciativa Emprendedora.

El documento se estructura de la siguiente forma: en el primer apartado, se hace una revisión de la literatura respecto al emprendedor y el emprendimiento, las características, las capacidades emprendedoras y la motivación por parte de la institución educativa, así como la teoría del Comportamiento Planificado, en la segunda parte, se presenta la metodología utilizada, seguido de los resultados $\mathrm{y}$ finalmente, se incluyen las conclusiones.

\section{Revisión de la literatura \\ Emprendedor y emprendimiento}

El INADEM (como se cito en Chan, Escalente y Robles, 2015) menciona que los emprendedores son personas con habilidades para lograr cambios en el entorno que lo rodea, generan cambios positivos a nivel individual, del sector, del país, continental o mundial, se enfocan en nichos de mercado con un alto nivel de estabilidad, en tendencias mundiales, los emprendedores impulsan proyectos con un amplio contenido de innovación, buscan trascender en el entorno que los rodea y ser una referencia del sector . Lo que realmente diferencia al empresario emprendedor es un proceso caracterizado por la innovación constante. Es por esto que, los trabajos recientes, proponen la importancia de considerar al proceso emprendedor como un conjunto de funciones, actividades y acciones asociadas con la percepción de oportunidad y la creación de la organización para explotarla.

Al respecto, Ortegón y Cardoso (2016) mencionan que un ingeniero debe orientarse a ser innovador y emprendedor para estar conectado con los cambios a los que hoy se enfrentan las organizaciones y la sociedad. Indican que para materializar sus ideas un ingeniero emprendedor debe realizar lo siguiente: identificar con qué recursos cuenta y lo que está dispuesto a hacer con ellos; ubicar donde se encuentra y cuál es el camino que debe trazar para llegar a donde planea; reconocer sus fortalezas y debilidades, valores y ganas 
de ser exitoso; ser capaz de actuar de manera independiente, sin presiones ni prejuicios de terceros; cambiar paradigmas a través de una mente abierta y dispuesto a hacer cosas diferentes; transformar sus ideas y descubrir nuevos talentos; mantener una alta capacidad de aprendizaje, aprendiendo y desaprendiendo; confiar en sí mismo, explotar su potencial motivaciónal para no renunciar a sus metas; aprovechar cada oportunidad de mejora continúa que se presente.

En los últimos años, el emprendimiento y el emprendedor han generado un fuerte interés como potencial generador de oportunidades y crecimiento profesional y económico.

El emprendimiento se puede definir como la capacidad de una persona para identificar oportunidades en su entorno, con la finalidad de alcanzar beneficios e impacto en la sociedad, para lo cual debe correr ciertos riesgos financieros (Sepúlveda, Denegri, Orellana, Criado, Mendoza, Salazar y Yung, 2017). Es así como el emprendimiento no solo traería beneficios personales, sino también sociales. Implica el descubrimiento, evaluación y explotación de oportunidades, la introducción de nuevos bienes y servicios, nuevas formas de organización y nuevos procesos y materiales (Delgado et al., 2008).

Históricamente el emprendimiento ha sido una forma de generar empleo y lograr un modelo de desarrollo socio-económico alternativo que incorpora y mejora el bienestar social de individuos excluidos del mercado laboral a pesar de contar una carrera universitaria (Rangel, Alvarado y Sierra, 2016). Es así como el emprendimiento se ha convertido en muchos países, en centro de atención, de estudios e investigaciones, motivando e incentivando a hacer empresa a través de diversos mecanismos generando no solo empleo e ingresos sino también innovación mediante la integración de universidades, comunidad y empresa; con el fin de generar escenarios de participación capaces de generar alternativas productivas y el fomento de una cultura emprendedora.

\section{Características de los emprendedores}

Las características de los emprendedores han sido ampliamente estudiadas por diversos investigadores, encontrando que el apoyo familiar respecto al emprendimiento, la motivación emprendedora por parte de la institución educativa, provenir de una familia con empresa, tener valores individualistas y ser líder, son consideradas como particularidades de los emprendedores, mientras que la edad, el género, el orden de nacimiento y tener experiencia emprendedora, no son variables significativas en la decisión de emprender (Lagunes, Herrera, González, y Hernández, 2013).

Según Chan et al. (2015) las características más señaladas de los emprendedores por diversos autores por ser las mencionadas y que se 
diferenciaban claramente unas de otras, fueron: creatividad, iniciativa, autoconfianza (confianza en sí mismo), energía y capacidad de trabajo, perseverancia, liderazgo, aceptación del riesgo, necesidad de logro, tolerancia al cambio y manejo de problemas. Lo anterior, resulta fundamental para identificar cuáles de estos factores pueden incidir en el emprendimiento de los estudiantes.

\section{Capacidades emprendedoras y motivación emprendedora por la institución educativa}

Cabana, Cortes, Plaza, Castillo y Alvárez (2013) al analizar las capacidades emprendedoras potenciales y efectivas en alumnos de nivel superior, identificaron cuatro factores determinantes: atributos del emprendedor, capacidades interpersonales, capacidades frente al riesgo y actitud del emprendedor, siendo este último factor el de mayor importancia junto con atributos del emprendedor representando ambos el $76 \%$, seguido de capacidad emprendedora potencial de $74 \%$ y alumnos con capacidad emprendedora efectiva solo un $16 \%$, los autores indican que al existir una gran brecha entre las capacidades emprendedoras potenciales y efectiva, produce un alto costo de oportunidad en los centros de educación superior, representando el desarrollo de capacidad emprendedora un desafío estratégico para cada uno de los centros de educación superior, para contribuir a su valor y complementariamente al desarrollo local, regional y nacional.

En cuanto a la motivación emprendedora, que tiene que ver con la vocación emprendedora, se relaciona con la valoración hacia el logro impulsada por la institución educativa donde los alumnos son formados, así lo mostraron Liseras, Gennero de Rearte y Graña (2004) al analizar la vocación emprendedora en alumnos del último año de tres carreras de una Universidad Argentina, obtuvieron que un $35 \%$ de los estudiantes tienen inclinaciones hacia actividades empresariales, es decir, vocación emprendedora, $20 \%$ con actitud emprendedora y $20.5 \%$ con intención emprendedora.

En otro estudio similar, se afirma que conforme el estudiante avanza en sus estudios la intención de crear su propia empresa va disminuyendo, esto es especialmente interesante, por la responsabilidad de una institución universitaria, en donde la actitud emprendedora no sólo se desarrolla a lo largo de la vida del estudiante, sino por el contrario el estudiante se desincentiva (Espiritu, 2011). Esto provocado por la educación que se les imparte en la universidad inclinada más hacia la gestión que hacia la creación de empresas.

Otro punto de vista se orienta en definir que los emprendedores no sólo nacen, se hacen (Sánchez, Caggiano, y Hernández, 2011). Es por esto 
que enseñar a emprender corresponde a la educación de los países que quieren mover el empleo fuera de lo público y de las grandes empresas. Destacan a la universidad como transmisora de conocimiento a la sociedad a partir del fomento del emprendimiento en sus estudiantes, dotándoles no solo de conocimientos sino también de habilidades y actitudes que les permitan transferir sus conocimientos en un producto económico y socialmente rentable.

En el mismo año, Pulgarín y Cardona (2011) mencionan que para el caso de los países desarrollados la motivación al emprendimiento surge en la oportunidad, pues con ingresos per cápita superiores pueden verse reflejados en inversión en vez de convertirse en ahorro por representar un mayor costo de oportunidad. En el caso contrario en los países no desarrollados la motivación se orienta hacia el lado de la necesidad. En otro estudio realizado en 3,359 alumnos universitarios y 807 alumnos de preparatoria del Tecnológico de Monterrey, se implementó un censo para detectar el grado de emprendimiento de los estudiantes y la composición empresarial del campus. Los resultados y hallazgos más relevantes a nivel profesional mostraron que $79 \%$ de los alumnos desean iniciar su propia empresa, es decir, un nivel muy alto de estudiantes potencialmente emprendedores. A su vez, observaron que el $80 \%$ de los alumnos de preparatoria persiguen esta misma meta (Lagunes et al., 2013).

Otro estudio similar fue desarrollado por Bridshaw, Bonomo, González, Herrera y Krauss (2014) quienes comparan tres universidades de México, Uruguay y Chile con diferente nivel de desarrollo, caracterizando los rasgos emprendedores de estudiantes de primer año, identificando la composición empresarial de las empresas de los estudiantes, su innovación y la relación que los alumnos manifiestan tener con la empresa familiar. Los resultados mostraron que el porcentaje de alumnos que desea iniciar su empresa vario de $76-90 \%$, lo cual demuestra el amplio potencial de emprendimiento en dichos estudiantes, a su vez la actitud emprendedora se reflejó en porcentajes de $54-71 \%$, dicho potencial se ve avalado por la posición del liderazgo que asumen los estudiantes y una alta valoración al logro.

Un año después, Chan et al. (2015) al comparar el nivel de emprendimiento de los alumnos de la carrera económico administrativas en dos universidades de Puerto Rico y México, en cuanto a las dimensiones de creatividad, seguridad, aceptación de riesgo y manejo de problemas, además de identificar las principales características que debe tener un emprendedor que inicia un negocio. Los hallazgos de mayor importancia fue la identificación de la dimensión la de aceptación del riesgo que establece diferencia entre estudios de dos universidades de diferentes países. 
Finalmente, al comparar las características emprendedoras personales y la alfabetización económica en estudiantes de dos facultades de una universidad pública ubicada en el sur de Chile por Sepúlveda et al. (2017), encontraron que los estudiantes de una de las facultades obtuvieron mayor puntuación en las siguientes dimensiones: anticipación del riesgo, persistencia y confianza, autoexigencia y calidad, búsqueda de control y exigencia, propositividad y previsión de futuro. Lo cual sugiere la tendencia a ser más flexible ante las demandas del entorno y a concentrarse en el cumplimiento de sus objetivos, a buscar la calidad en todo ámbito tanto como la exige de sí mismo. Es decir, una persona con este perfil tiende a planificar y a basar sus decisiones en función de los beneficios y costos de sus actos. Los resultados eran esperados debido a que dichos son formados para desarrollar actitudes y competencias favorables hacia el emprendimiento.

\section{Teoría del comportamiento planificado}

Esta teoría identifica actitudes hacia el comportamiento, normas subjetivas y control de comportamiento percibido, los cuales en conjunto determinan la intención de crear una empresa y finalmente concretarse en la creación de nuevas unidades productoras. De acuerdo con Ajzen (1991), en la teoría del comportamiento planificado, los recursos y las oportunidades que están a disposición de una persona dictan de alguna manera la probabilidad de manifestar un determinado comportamiento. Las actitudes, las normas subjetivas y el control conductual percibido están relacionados con conjuntos apropiados de creencias conductuales, normativas y de control relevantes sobre el comportamiento, pero la naturaleza exacta de estas relaciones aún es incierta. De igual forma, es importante precisar, que la teoría del comportamiento planificado ha sido utilizada en diferentes estudios, entre estos se ha evaluado las actitudes al conducir y los intentos por predecir el comportamiento infractor en conductores (Moyano, 1997). Es así que tomando como enfoque del presente estudio la teoría mencionada, es preciso definir que la intención emprendedora es considerada una área consolidada de investigación con una rápida evolución y un número creciente de investigaciones, con el objetivo de identificar las tendencias y la manera en que ha evolucionado el estudio de la intención emprendedora en general y en estudiantes universitarios (Valencia, Montoya y Montoya, 2016), atrayendo el interés de investigadores, provocando así la generación de conocimientos de manera progresiva (Liñán y Fayolle, 2015).

Las intenciones de crear un negocio se han analizado habitualmente en alumnos de diferentes países. Muchos de los trabajos han abordado el estudio de las intenciones como buen predictor del comportamiento de los individuos centrado su atención en los estudiantes (Delgado et al., 2008). La 
mayor parte de ellos en contextos distintos al latinoamericano sobresaliendo las universidades con carreras administrativas o creación de empresas. Entre estas investigaciones Espiritu (2011) determinó la importancia que tienen los rasgos psicológicos de personalidad riesgo, necesidad de logro, locus de control interno y propensión al riesgo en la intención emprendedora de estudiantes universitarios de administración y contador público de la Universidad de Colima. El resultado mostró una alta relación positiva significativa de los tres rasgos de personalidad con respecto a su intención de crear su propia empresa por parte de los estudiantes. Los factores que inciden en la intención emprendedora de los estudiantes de las áreas económicoadministrativas de un centro universitario varían de acuerdo con el contexto a partir de creencias, actitud hacia la conducta, las intenciones y la conducta en sí (Jaimes, Jamarillo y Pérez, 2017).

Por su parte, De Jorge (2013) analizó los factores que influyen en la intención emprendedora en los estudiantes universitarios de los últimos cursos de la licenciatura en administración y dirección de empresas. Los resultados principales mostraron que las características personales (sociodemográficas) de los alumnos en términos de mayor edad, género masculino y la existencia de algún familiar empresario han resultado ser explicativas de la intención emprendedora.

La intención emprendedora se considera un requisito previo de la decisión de emprender un negocio. Las variables demográficas, la capacidad percibida y la educación para emprender ayudan a explicar la intención emprendedora, así lo demuestra Soria-Barreto, Zuniga-Jara y Ruiz-Campo (2016) tras analizar en estudiantes universitarios cómo un curso de emprendimiento puede afectar la intención emprendedora de los estudiantes. Mostraron que la intención emprendedora fue afectada negativamente por el curso. A su vez los estudiantes que experimentaron el mayor cambio en su voluntad de emprender fueron quienes poseían dos características: consideraban tener los conocimientos, habilidades y experiencia para poner en marcha un nuevo negocio y pertenecían a familias de menores ingresos de renta familiar. Es así, como a partir de la revisión de la literatura, se plantea la siguiente hipótesis:

$\mathrm{H}_{1}$ : Existe asociación entre la intención de elegir la carrera al término de sus estudios y el grado de Iniciatiava Emprendedora.

\section{Metodología}

De acuerdo con la revisión de la literatura y la importancia que el Tecnológico Nacional de México (2017) señala como objetivo de la carrera de Ingeniería Civil: formar ingenieros civiles de manera integral, con visión humana, analítica, creativa y emprendedora que contribuyan al desarrollo de 
la sociedad, definiendo claramente la necesidad de conocer el nivel de desarrollo de los factores que constituyen el Grado de Intención Emprendedora de los estudiantes.

La investigación se define como básica orientada, de enfoque cuantitativo, alcance descriptivo y relacional. En función del proyecto de investigación descrito y su objetivo, la unidad de análisis son los estudiantes de los ocho semestres del programa educativo de Ingeniería Civil del Instituto Tecnológico Superior del Oriente del Estado de Hidalgo, perteneciente al Tecnológico Nacional de México, el cual cuenta con una población de 408 estudiantes, por lo que se procedió a realizar el cálculo de la muestra estadística, considerando un error del 5\% y confiabilidad del 95\% resultando un total de 198 estudiantes encuestados, quienes fueron invitados a responder la encuesta en las aulas de la institución.

Como instrumento para la recolección de la información se aplicó la adaptación del GUESSS (Global University Entrepreneurial Spirit Student's Survey), el cual tiene como propósito comprender la intención y la actividad emprendedora de los estudiantes universitarios (Medina, Bolívar y Lemes, 2014). El instrumento consta de dos apartados: la primera parte, evaluó variables sociodemográficas como: género, edad, estado civil y semestre. La segunda sección, se integró por preguntas de respuesta cerrada, de acuerdo con lo siguiente: cuatro preguntas con opción múltiple que midieron la elección de la carrera universitaria con enfoque hacia el pasado y futuro; seis preguntas de respuesta binaria que midieron antecedentes y formación en materia de emprendimiento; así como 18 ítems que evaluaron cuatro factores del perfil emprendedor: intención emprendedora, actitud personal hacia el emprendimiento, control del comportamiento percibido y normas subjetivas, las cuales se midieron mediante la escala tipo Likert, de acuerdo con lo siguiente: 1= Muy en desacuerdo, 2=En desacuerdo, 3=Neutral, 4=De acuerdo 5=Muy de acuerdo.

Con el alcance descriptivo, se obtienen las características específicas de los estudiantes, como: género, edad, estado civil y semestre, así como las puntuaciones medias por factor que determina la Iniciativa Emprendedora. Después se procedió a realizar el cálculo del Grado de Iniciativa Emprendedora, considerando tres opciones, bajo, medio y alto. Finalmente, para contrastar la hipótesis, se aplicó la prueba estadística de Chi-cuadrado con la intención de asociación las variables planteadas en la hipótesis.

\section{Resultados}

Como parte del análisis descriptivo, a continuación, se presentan los resultados de las variables sociodemográficas, mostrándose en la Tabla 1, en cuanto al género se observa que el $77.8 \%$ son hombres, representando la mayoría, esto se puede deber, a que tradicionalmente la carrera de Ingeniería 
Civil la estudia en su mayoría el género masculino. Respecto al estado civil, se obtuvo que la mayoría son solteros y en su mayoría tienen entre 19 y 20 años, siendo congruente en virtud, de que a esa edad no asumen otro tipo de compromiso. Por último, referente al semestre en que se encuentran inscritos los encuestados, la mayoría manifiesta pertenecer al octavo semestre, siendo esto fundamental debido a que son próximos a egresar y el emprendimiento puede ser una oportunidad de carrera.

Tabla 1. Variables sociodemográficas

\begin{tabular}{|c|c|c|c|}
\hline Variable & Descripción & Número & Porcentaje \\
\hline \multirow{3}{*}{ 엄 } & Masculino & 154 & 77.8 \\
\hline & Femenino & 44 & 22.2 \\
\hline & Total & 198 & 100 \\
\hline \multirow{5}{*}{ 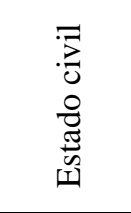 } & Soltero & 177 & 89.4 \\
\hline & Casado & 11 & 5.6 \\
\hline & Unión Libre & 7 & 3.5 \\
\hline & Otro & 3 & 1.5 \\
\hline & Total & 198 & 100 \\
\hline \multirow{6}{*}{ 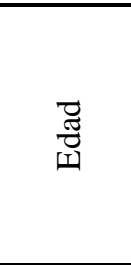 } & Hasta 18 años & 14 & 7.1 \\
\hline & 19-20 años & 84 & 42.4 \\
\hline & 21-22 años & 62 & 31.3 \\
\hline & 23-24 años & 29 & 14.6 \\
\hline & 25 años o más & 9 & 4.5 \\
\hline & Total & 198 & 100 \\
\hline \multirow{9}{*}{ 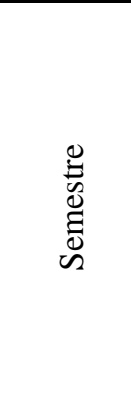 } & Primero & 21 & 10.6 \\
\hline & Segundo & 28 & 14.1 \\
\hline & Tercero & 20 & 10.1 \\
\hline & Cuarto & 33 & 16.7 \\
\hline & Quinto & 13 & 6.6 \\
\hline & Sexto & 28 & 14.1 \\
\hline & Séptimo & 19 & 9.6 \\
\hline & Octavo & 36 & 18.2 \\
\hline & Total & 198 & 100 \\
\hline
\end{tabular}

Fuente: Elaboración propia

Por otra parte, en la Tabla 2 y Figura 1, se presentan los resultados de la intención de elección de la carrea de Ingeniería Civil, se observa que tanto al inicio de la carrera como cinco años después, la mayoría de los estudiantes aspira a ser fundador de su propia empresa, igualmente se identifica que la intención emprendedora incrementa en $14.1 \%$ entre la elección al término de los estudios y cinco años después. Los resultados, son similares a los obtenidos por Medina, Bolívar y Lemes (2014) quienes en su estudio indican que la intención emprendedora de los estudiantes encuestados se incrementa cinco años después de terminar su carrera. 
Tabla 2. Elección de la carrera al termino de los estudios y cinco años después

\begin{tabular}{cccccc}
\hline $\begin{array}{c}\text { Intención de la elección } \\
\text { de la carrera de } \\
\text { Ingeniería Civil }\end{array}$ & $\begin{array}{c}\text { Ser } \\
\text { empleado }\end{array}$ & $\begin{array}{c}\text { Ser fundador } \\
\text { de mi propia } \\
\text { empresa }\end{array}$ & $\begin{array}{c}\text { Ser sucesor } \\
\text { de una } \\
\text { empresa }\end{array}$ & $\begin{array}{c}\text { Otro, no lo } \\
\text { sé todavía }\end{array}$ & Total \\
\hline $\begin{array}{c}\text { ¿Cuál es su intención de } \\
\text { elegir la carrera, al } \\
\text { término de sus } \\
\text { estudios? }\end{array}$ & 16.7 & 67.7 & 4.5 & 11.1 & 100 \\
$\begin{array}{c}\text { ¿Cuál es su intención de } \\
\text { elegir la carrera, cinco } \\
\text { años después del } \\
\text { término de sus } \\
\text { estudios? }\end{array}$ & 9.1 & 81.8 & 6.1 & 3.0 & 100 \\
\hline
\end{tabular}

Fuente: Elaboración propia

¿Cuál es su intención de elegir la carrera,

cinco años después del término de sus estudios?

¿Cuál es su intención de elegir la carrera, al término de sus estudios?

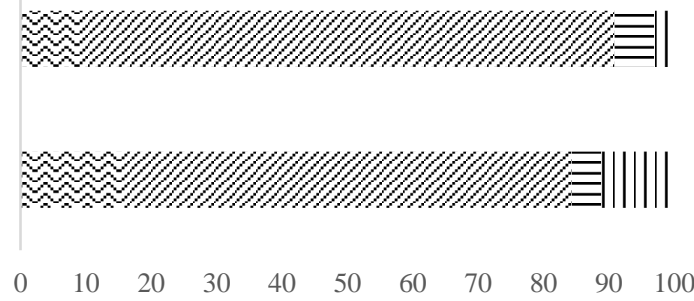

$\lessdot$ Ser empleado

थ. Ser fundador de mi propia empresa

= Ser sucesor de una empresa.

IIOtro, no lo sé todavía.

Figura 1. Elección de la carrera al termino de los estudios y cinco años después

Fuente: Elaboración propia

A continuación, en la Tabla 3 y Figura 2, se presentan los resultados de las puntuaciones medias de los factores que respecto a la Teoría del Comportamiento Planificado se consideran en el perfil emprendedor que potencializa la iniciativa emprendedora, los datos indican que de manera general el Grado de Iniciativa Emprendedora, se ubica en 3.9787, mientras que los factores se presentan en el siguiente orden: intención emprendedora (4.2163), actitud personal hacia el emprendimiento (4.0020), normas subjetivas (3.8973) y por último el control de comportamiento percibido (3.7992). Los datos anteriores indican que el factor que más se presenta en los estudiantes es la intención emprendedora que se refiere a la idea que tienen de iniciar o desarrollar una idea de negocio en un futuro. Por otra parte, la puntuación más baja se obtuvo en el control de comportamiento percibido, que se refiere al grado que asume el estudiante para desarrollar el comportamiento específico de emprender. 
Tabla 3. Factores de la Intención Emprendedora

\begin{tabular}{cccccc}
\hline Factor & $\mathrm{N}$ & Media & Mínimo & Máximo & $\begin{array}{c}\text { Desviación } \\
\text { estándar }\end{array}$ \\
\hline $\begin{array}{c}\text { 1.Intención } \\
\text { Emprendedora }\end{array}$ & 198 & 4.2163 & 1.1667 & 5.0000 & 0.6258 \\
$\begin{array}{c}\text { 2.Actitud Personal } \\
\text { hacia el }\end{array}$ & 198 & 4.0020 & 1.6000 & 5.0000 & 0.6613 \\
$\begin{array}{c}\text { Emprendimiento } \\
\text { 3.Control de }\end{array}$ & 198 & 3.7992 & 2.2500 & 5.0000 & 0.5938 \\
$\begin{array}{c}\text { Comportamiento } \\
\text { Percibido }\end{array}$ & 198 & 3.8973 & 1.6667 & 5.0000 & 0.6851 \\
$\begin{array}{c}\text { 4.Normas Subjetivas } \\
\text { 5.Grado de iniciativa } \\
\text { emprendedora }\end{array}$ & 198 & 3.9787 & 2.6500 & 5.0000 & 0.4747 \\
\hline
\end{tabular}

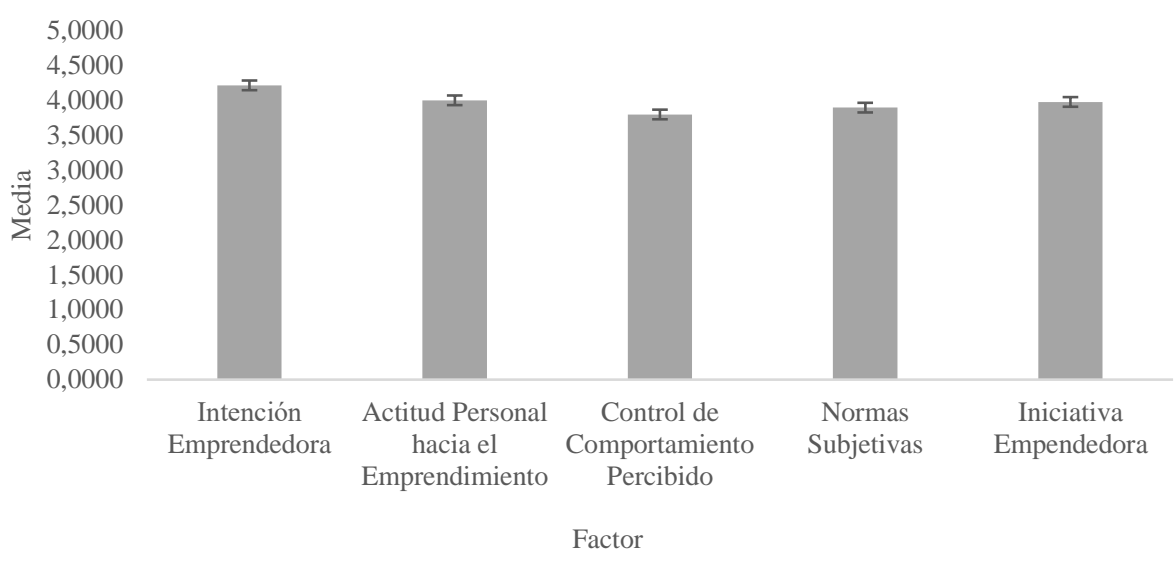

Figura 2. Factores de la intención emprendedora Fuente: Elaboración propia

Con la finalidad de contrastar la hipótesis de investigación planteada, a continuación, se procede a realizar la prueba estadística de Chi-cuadrada, la cual se aplica, debido a que las dos variables consideradas como parte de la hipótesis son categóricas, correspondiente a la intención de elección de carrera al término de los estudios y el Grado de Iniciativa Emprendedora.

Los resultados se presentan en el siguiente orden: $67.7 \%$ tienen intención de fundar su propia empresa, $16.7 \%$ opina que serán empleados, el $11.1 \%$ todavía no sabe que harán al término de sus estudios y, el $9 \%$ de los encuestados prevé que serán sucesores de una empresa. De igual forma, los datos muestran que respecto al grado de iniciativa emprendedora, 58 estudiantes tienen un grado medio y 140 un alto grado.

Los números indican que de los estudiantes aspirantes a ser fundadores de su empresa el 55.2\% tiene un grado de iniciativa emprendedora medio y $72.9 \%$ alto. Respecto a la prueba estadística (Tabla 4) 
se indica que el porcentaje de celdas, con un recuento esperado menor que 5, es del $12.5 \%$, por lo tanto, como el valor del estadístico de contraste (observado) es 9.682 , el cual muestra, en la distribución 3 grados de libertad $(\mathrm{gl}=3)$, así como una significación asintótica de $(0.021)$, por lo que el valor de $p$, es menor que 0.050 , lo cual permite aceptar la hipótesis de dependencia que indica que la intención de elegir la carrera al término de sus estudios en los estudiantes de Ingeniería Civil se relaciona con el grado de iniciativa emprendedora. Los resultados, dan oportunidad para generar nuevas líneas de investigación, dado que existe carencia de estudios específicos en el análisis de esta carrera, lo cual pudiera ser considerado como área de oportunidad, dado que los retos de la ingeniería y las políticas públicas del país hoy en día se orientan al fomento del emprendimiento, lo cual ya no es específico en las áreas administrativas, de lo cual existen muchos estudios.

Tabla 4. Prueba de Chi-cuadrada de la intención de elección de la carrera al término de los estudios y el grado de iniciativa emprendedora

\begin{tabular}{|c|c|c|c|c|c|}
\hline & \multicolumn{2}{|c|}{ Descripción } & \multicolumn{2}{|c|}{$\begin{array}{l}\text { Grado de Iniciativa } \\
\text { Emprendedora }\end{array}$} & \multirow[t]{2}{*}{ Total } \\
\hline & & & Medio & Alto & \\
\hline \multirow{15}{*}{ 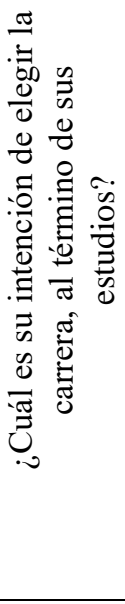 } & Ser empleado & Recuento & 10 & 23 & 33 \\
\hline & & Recuento esperado & 9.7 & 23.3 & 33.0 \\
\hline & & $\%$ dentro de GIE & $17.2 \%$ & $16.4 \%$ & $16.7 \%$ \\
\hline & Ser fundador & Recuento & 32 & 102 & 134 \\
\hline & de mi propia & Recuento esperado & 39.3 & 94.7 & 134.0 \\
\hline & empresa & $\%$ dentro de GIE & $55.2 \%$ & $72.9 \%$ & $67.7 \%$ \\
\hline & Ser sucesor de & Recuento & 4 & 5 & 9 \\
\hline & una empresa. & Recuento esperado & 2.6 & 6.4 & 9.0 \\
\hline & & $\%$ dentro de GIE & $6.9 \%$ & $3.6 \%$ & $4.5 \%$ \\
\hline & Otro, no lo sé & Recuento & 12 & 10 & 22 \\
\hline & todavía. & Recuento esperado & 6.4 & 15.6 & 22.0 \\
\hline & & $\%$ dentro de GIE & $20.7 \%$ & $7.1 \%$ & $11.1 \%$ \\
\hline & Total & Recuento & 58 & 140 & 198 \\
\hline & & Recuento esperado & 58.0 & 140.0 & 198.0 \\
\hline & & $\%$ dentro de GIE & $100.0 \%$ & $100.0 \%$ & $100.0 \%$ \\
\hline
\end{tabular}

...Tabla 4. Prueba de Chi-cuadrada de la intención de elección de la carrera al término de los estudios y el grado de iniciativa emprendedora.

1 casillas $(12.5 \%)$ han esperado un recuento menor que 5 . El recuento mínimo esperado es 2.64 . 


\section{Agradecimientos}

Agradecemos al Tecnológico Nacional de México y al Instituto Tecnológico Superior del Oriente del Estado de Hidalgo (ITESA), por el apoyo otorgado para la realización del presente estudio.

\section{Conclusion}

En la actualidad, el emprendimiento se ha convertido en una oportunidad deseable de carrera de los futuros profesionistas, siendo uno de los retos de la Academia de Ingeniería y específicamente de las Instituciones de Educación Superior (IES), trabajar en desarrollar y fortalecer la iniciativa emprendedora como oportunidad de crecimiento profesional y económico.

Con la presente investigación, se ha logrado el objetivo de analizar la iniciativa emprendedora de los estudiantes de Ingeniería Civil en una IES en el estado de Hidalgo. Así mismo, resulta importante mencionar, que derivado de los resultados obtenidos, en mayor porcentaje los estudiante eligen la carrera con la intención de emprender tanto al término de sus estudios como cinco años después, aunque es claro el aumento de la iniciativa tiempo después, lo cual es una oportunidad para la institución de establecer estrategias que fortalezcan el potencial emprendedor, dado que la estructura curricular no cuenta formalmente con asignaturas, sin embargo, recientemente en la institución se está implementando el fortalecer emprendedores desde los primeros semestres de la formación.

En cuanto a los factores de la teoría analizada, se presentaron en el siguiente orden: intención emprendedora, actitud personal hacia el emprendimiento, normas subjetivas $\mathrm{y}$ por último el control de comportamiento percibido, lo cual da pauta para implementar efectivamente la estrategia del fomento y acompañamiento de expertos durante el desarrollo de la idea empresarial y formular talleres que fortalezcan la seguridad para el control de comportamiento percibido y desarrollar una mentalidad de retos en los futuros profesionistas para consolidar el emprendimiento.

De igual forma se concluye que la mayoría de los estudiantes tienen intención de emprender su propia empresa, teniendo en su mayoría un grado de emprendimiento alto, por lo cual, se puede definir que la intención de elegir la carrera al término de sus estudios en los estudiantes de Ingeniería Civil se relaciona con el grado de iniciativa emprendedora, por lo cual se acepta la hipótesis planteada.

Lo anterior, permite desarrollar estrategias para fomentar el emprendimiento y enfocarse en las dimensiones que pueden afectar la intención de emprender por parte de los futuros ingenieros.

La principal limitación de la investigación está en la aplicación en una sola IES, por lo que es necesario incrementar la muestra en otras instituciones. 


\section{References:}

1. Ajzen, I. (1991). The Theory of Planned Behavior. Organizational Behavior and Human Decision Processes, 50,179-211

2. Bridshaw, A. L., Bonomo, O. A., González, T. L., Herrera, A. M., y Krauss, D. C. Estudio comparativo de emprendimiento y empresa familiar en estudiantes universitarios de México, Uruguay y Chile. Recuperado de https://cladea2014.files.wordpress.com/2014/10/paper_277.pdfCaban a-Villca, R., Cortes

3. Cabana, V. R., Cortes, C. I., Plaza, P. D., Castillo, V. M., y Alvárez M. A. (2013). Análisis de las capacidades emprendedoras potenciales y efectivas en alumnos de Centros de Educación Superior. J. Technol. Manag. Innov., 8(1),65-75

4. Consejo de la Acreditación de la enseñanza de la Ingeniería A.C. CACEI. (2016). Recuperado de http://www.cacei.org.mx/nvfs/nvfs04/nvfs040301.php

5. Chan, M. M. R., Escalante, M. N. del S., y Robles, M. E. (2015). Comparabilidad en el nivel de emprendimiento de los alumnos de dos universidades: México y Puerto Rico. Revista Internacional Administración y Finanzas, 8(5),1-14

6. De Jorge, M. J. (2013). Análisis de los factores que influyen en la intención emprendedora de los estudiantes universitarios. Caracciolos, 1(1),2-12

7. Delgado, P. M. I., Gómez, M. L., Romero, M. A. M., y Vázquez, I. E. (2008). Determinantes sociales y cognitivos en el espíritu emprendedor: un estudio exploratorio entre estudiantes argentinos. Cuadernos de Gestión, 8(1),11-2

8. Espíritu, O. R. (2011). Análisis de la intención emprendedora en estudiantes universitarios a través de los rasgos de personalidad. Multiciencias, 11(1),65-75

9. Jaime, P. A., Gómez, M. M., y Ayala, M. G. (2016). La ingeniería civil mexicana, estado actual y acciones para enfrentar los retos del siglo XXI. Academia de Ingeniería de México. Recuperado de http://www.ai.org.mx/sites/default/files/la_ingenieria_civil_mexicana _estado_actual.pdf

10. Jaimes, M. F., Jamarillo, J. M., y Pérez, Ch. M. A. (2017). Factores que inciden en la intención emprendedora de estudiantes del Centro Universitario Tempascaltepec. Revista Venezolana de Gerencia, 22 (78), 210-231

11. Kantis, H. Federico, J. e Ibarra, S. (2014). Condiciones sistémicas para el emprendimiento dinámico 2016: novedades y tendencias para fortalecer e integrar los ecosistemas de la región. Recuperado de: 
http://www.ungs.edu.ar/icsedprodem/wpcontent/uploads/2015/07/informe-completo-FINAL_2016.pdf

12. Lagunes, T. L. A., Herrera, A. M., González, T. L. A., y Hernández, R. K. E. (2013). Censo 2013. Espíritu emprendedor y familias emprendedoras. Tecnológico de Monterrey, Campus Puebla. Recuperado de https://www.editorialdigitaltec.com/materialadicional/Publicacioncen so2013.pdf

13. Liseras, N., Gennero de Rearte, A., y Graña, F. (2004). Vocación emprendedora en la Universidad Argentina. Recuperado de https://www.uv.es/motiva/libromotiva/13LiserasGenneroGraña.pdf

14. Liñán, F., y Fayolle, A. (2015). A systematic literature review on entrepreneurial intentions: Citation, thematic analyses, and research agenda. International Entrepreneurship and Management Journal, 11(4), 907-933. Doi: 10.1007/s11365-015-0356-5

15. Medina, P., Bolívar, A. y Lemes, A.I. (2014). Un paso más en la investigación de la intención emprendedora del estudiante universitario: GUEESS. Revista de Estudios Empresariales, 2, 63-80.

16. Moyano, D. E. (1997). Teoría del Comportamiento Planificado e intención de infringir normas de tránsito en peatones. Estudios de Psicología, 2(2),335-348

17. Ortegón, A. M. L. I., y Cardoso, L. G. (2016). Importancia del emprendimiento en la formación integral del ingeniero en México. Revista Electrónica ANFEI Digital, 2(5),1-8

18. Pulgarín, M. S. A. y Cardona, A. M. (2011). Caracterización del comportamiento emprendedor para los estudiantes de administración de la Universidad del Rosario. Revista Escuela de Administración de Negocios, 71,22-39

19. Rangel, L. O., Alvarado, M. M., y Sierra, O. E. (2016). El perfil emprendedor como catalizador de la innovación en los jóvenes del Departamento de la Guajira. Omnia, 22(3),71-86

20. Sánchez, J. C., Caggiano, V., y Hernández, V. (2011). Competencias emprendedoras en la educacion universitaria. International Journal of Developmental and Educational Psychology, 3(1),19-28

21. Secretaría de Economia. (2017). Recuperado de http://www.20062012.economia.gob.mx/mexico-emprende/empresas/emprendedor

22. Tecnológico Nacional de México TNM. (2017). Ingeniería Civil. Recuperado de http://www.tecnm.mx/licenciatura_2009_2010/ingenieria-civil

23. Sepúlveda, M. J., Denegri, C. M., Orellana, C. L., Criado, N., Mendoza, J., Salazar, P., y Yung, G. (2017). Características emprendedoras personales y alfabetización económica: Una 
comparación entre estudiantes universitarios del sur de Chile. Interdisciplinaria, 34(1), 107-124

24. Soria-Barreto, B. K., Zuniga-Jara, S., y Ruiz-Campo, S. (2016). Educación e intención emprendedora en estudiantes universitarios: Un caso de estudio. Formación universitaria, 9(1),25-34

25. Valencia, A. A., Montoya, R. I., y Montoya, R. A. (2016). Intención emprendedora en estudiantes universitarios: Un estudio bibliométrico. Intangible Capital, 12(4),881-922 